\title{
Determinants of uncontrolled dyslipidaemia among adult type 2 diabetes in Malaysia: The Malaysian Diabetes Registry 2009.
}

\begin{abstract}
Numerous studies with compelling evidence had shown a clear relationship between dyslipidaemia and cardiovascular (CV) events in patients with diabetes mellitus. This was an observational study based on secondary data from the online registry database Adult Diabetes Control and Management (ADCM) looking into the determinants of uncontrolled dyslipidaemia in type 2 diabetes mellitus patients. Independent predictors were identified using multivariate logistic regression. A total of 303 centres ( 289 health clinics, 14 hospitals) contributed a total of 70,889 patients (1972 or $2.8 \%$ patients were from hospital). About thirty eight percent were reported to have dyslipidaemia. There were $40.7 \%$ patients on lipidlowering agents and of those above age 40 years old, only $38.1 \%$ of them were on a statin. Malay ethnicity and younger age groups ( $<50$ years old) were two major determinants of uncontrolled LDL-C, TG and HDL-C. Female gender and uncontrolled blood pressure were determinants of uncontrolled LDL-C, and poor glycaemic control was related independently to high TG. This study has highlighted the suboptimal management of diabetic dyslipidaemia in Malaysia. Pharmacological treatment of dyslipidaemia could be more effective. Healthcare stakeholders in this country, especially in the primary care, have to recognize these shortfalls and take immediate remedial measures.
\end{abstract}

Keyword: Dyslipidaemia; HMG-CoA statins; LDL lipoproteins; Primary care; Type 2 diabetes mellitus. 